\title{
The average specific forced radiation wave impedance of a finite rectangular panel ${ }^{\text {a) }}$
}

\author{
John L. Davy ${ }^{\text {b) }}$ and David J. Larner \\ School of Applied Sciences, RMIT University, GPO Box 2476V Melbourne, Victoria 3001, Australia \\ Robin R. Wareing and John R. Pearse \\ University of Canterbury, Mechanical Engineering, Private Bag 4800, Christchurch 8140, New Zealand
}

(Received 4 March 2014; revised 20 June 2014; accepted 29 June 2014)

\begin{abstract}
The average specific forced radiation wave impedance of a finite rectangular panel is of importance for the prediction of both sound insulation and sound absorption. In 1982, Thomasson published numerical calculations of the average specific forced radiation wave impedance of a square of side length $2 e$ for wave number $k$ in half octave steps of $k e$ from 0.25 to 64 . Thomasson's calculations were for the case when the forced bending wave number $k_{b}$ was less than or equal to $k$. Thomasson also published approximate formulas for values of $k e$ above and below the published results. This paper combines Thomasson's high and low frequency formulas and compares this combined formula with Thomasson's numerical calculations. The real part of the approximate formula is between $0.7 \mathrm{~dB}$ higher and $-1 \mathrm{~dB}$ lower than the numerical calculations. The imaginary part of the approximate formula is between $2.3 \mathrm{~dB}$ higher and $-2.6 \mathrm{~dB}$ lower than the numerical calculations. This paper also gives approximate formulas for the case when $k_{b}$ is greater than or equal to $k$. The differences are between 0.8 and $-1.2 \mathrm{~dB}$ for the imaginary part and between 6.2 and $-2.4 \mathrm{~dB}$ for the real part. (C) 2014 Acoustical Society of America. [http://dx.doi.org/10.1121/1.4889866]
\end{abstract}

PACS number(s): 43.20.Rz, 43.40.Rj, 43.55.Rg, 43.55.Dt [MV]

Pages: 525-536

\section{INTRODUCTION}

The average specific radiation wave impedance of one side of a finite rectangular panel mounted in an infinite rigid baffle is of importance for the prediction of sound insulation, sound absorption and sound scattering. It occurs naturally when variational techniques are used to solve these phenomena (Thomasson, 1980, 1982; Allard and Atalla, 2009; Brunskog, 2012; Jeong, 2013). Thus, this average value can be viewed as the one sided specific radiation wave impedance fluid loading on a two dimensional transverse velocity wave which is propagating on a finite plane surface mounted in an infinite rigid baffle. The specific radiation wave impedance is the ratio of the radiated complex number sound pressure at a point on the surface of a radiating panel to the complex number transverse velocity of the panel at the same point. Because the specific radiation wave impedance will vary with position on the finite rectangular panel, the average is taken over the radiating surface of the panel. The specific radiation wave impedance may also vary with the azimuthal angle of propagation of the transverse velocity wave in the finite rectangular panel and in many situations the average will also be taken over azimuthal angle. For a transverse velocity wave in the panel which is forced by an incoming sound wave, the average for diffuse field excitation is also of interest.

\footnotetext{
${ }^{a}$ Portions of this work were presented in "The specific forced radiation wave impedance of a finite rectangular panel excited by a plane sound wave," the 21st International Congress on Sound and Vibration, Beijing, China, 13-17 July 2014.

b) Author to whom correspondence should be addressed. Current address: CSIRO Materials Science and Engineering, P.O. Box 56, Highett, Victoria 3190, Australia. Electronic mail: john.davy@rmit.edu.au
}

Thomasson (1982) published numerical calculations of the average specific forced radiation wave impedance of a square of side length $2 e$ for a forcing sound wave number $k$ in half octave steps of $k e$ from 0.25 to 64 and in $15^{\circ}$ steps of the incident angle of the forcing sound wave from $0^{\circ}$ to $90^{\circ}$. Thomasson (1982) also published approximate formulas for values of $k e$ above and below his published numerical results. In this case, the bending wave number $k_{b}$ of the forced transverse velocity wave propagating in the finite rectangular panel is less than the wave number $k$ of sound in the medium into which the panel is radiating. Thomasson's numerical results and his approximate formulas for a square are given in Table I of Thomasson (1982). Because this publication of Thomasson can be hard to obtain, Thomasson's Table I is reproduced as Table I in Jeong (2013). Note that the $e$ used by Thomasson and Jeong is the length of the side of the square and is thus twice the value of the $e$ used in this paper which is the half length of the side of the square. Thomasson's and Jeong's imaginary parts of the impedance are the opposite sign to the imaginary parts of the impedance in this paper because of their choice of a different complex sinusoidal variation with time than that used in this paper.

The real part of average specific radiation wave impedance of a panel, normalized by being divided by the characteristic impedance of the medium into which it is radiating, is equal to the radiation efficiency of the panel. There have been a number of authors who have studied the forced radiation efficiency of a finite rectangular panel. Gösele (1953) derived the radiation efficiency for a finite panel. He also included panel wavelengths which are less than the wavelength of the sound in the medium for which the infinite panel model predicts zero radiation efficiency. He gave approximate formulas for certain ranges of parameters and 
graphed results of numerical calculations for three different sizes of panels. Sato (1973) gave the results of much more extensive numerical calculations in both tabular and graphical form for the forced wave case where the panel wavelength is longer than the wavelength in air. Sato also numerically calculated the diffuse field forced radiation efficiency averaged over all possible directions of sound incidence.

Rindel (1975) used Sato's numerical results for the forced radiation efficiency in his theory of sound insulation as a function of angle of incidence and gave Sato's results in an English language publication. According to Novak (1992), Lindblad (1973) provided an approximate formula for the radiation efficiency at high frequencies based on Gösele's results. Lindblad (1985) gave a simpler approximation which could be integrated over all angles of incidence. He also extended the integrated formula to low frequencies. Rindel (1993a) presented a slightly more complicated version of the formula from Lindblad (1973), with constants which are selected to provide good agreement with Sato's tabulated radiation efficiencies. Rindel's formula also extends Lindblad's formula to low frequencies. This formula of Rindel is too complicated to be integrated easily by analytic means. However Rindel (1993b) gave an approximate formula for the diffuse field forced radiation efficiency. Ljunggren (1991) repeated Sato's numerical calculations using a two dimensional model and obtained agreement "well within $0.5 \mathrm{~dB}$ " for both as a function of angle of incidence and averaged over all angles of incidence. Novak (1995) performed even more extensive three dimensional calculations than Sato. Davy (2009) gave even better approximations for the forced radiation efficiency which could also be analytically integrated to calculate the diffuse field forced radiation efficiency. Davy also extended most of the previous models so that they covered the whole frequency range and compared them with Sato's numerically calculated values for the forced radiation efficiency. Approximate formulas for the radiation efficiency of a panel with freely propagating waves were derived by Lyon and Maidanik (1962), Maidanik (1962, 1975) and Leppington et al. (1982). None of the above papers include approximate formulae for the imaginary part of the average specific radiation wave impedance which cover the whole range of parameters.

The aim of this paper is to combine and extend the approximate equations derived by Thomasson (1982) so that they can also be used in the range covered by his numerically calculated and tabulated values which is not covered by his approximate formulas. The results given by these combined and extended equations are compared to the tabulated values of Thomasson (1982). The results of Thomasson (1982) are extended by the development of approximate formulae for the case where $k_{b}$ is greater than $k$. These approximate formulas are compared to numerically calculated values.

In this paper, the sinusoidal variation with time is assumed to be proportional to $e^{j \omega t}$, where $\omega$ is the angular frequency, $t$ is the time, $j$ is the square root of -1 and $e$ is Euler's number. $e$ is also used to define half the typical distance across the panel [see Eq. (41)], but this should not create any confusion. It should be noted that the assumption of $e^{-j \omega t}$ for the sinusoidal variation with time gives the opposite sign for the imaginary part of the impedance. The impedances in this paper are normalized by dividing by the characteristic impedance of the fluid medium $Z_{c}$, which is the product of the ambient density of the fluid medium $\rho_{0}$ and the speed of sound in the fluid medium $c$.

The geometry considered in this paper is shown in Fig. 1. An infinite one dimensional (either forced or unforced) sinusoidal bending wave with bending wave number $k_{b}$ traveling in an infinite isotopic panel immersed in a fluid medium with freely propagating wave number $k$ has a one sided normalized specific radiation wave impedance $z$ given by (Cremer et al., 2005)

$$
z= \begin{cases}1 / \sqrt{1-\left(k_{b} / k\right)^{2}}=1 / \sqrt{1-\mu^{2}}=1 / \cos (\theta) & \text { if } k_{b}<k \\ \infty & \text { if } k_{b}=k \\ j / \sqrt{\left(k_{b} / k\right)^{2}-1}=j / \sqrt{\mu^{2}-1} & \text { if } k_{b}>k,\end{cases}
$$

where

$$
\mu=\frac{k_{b}}{k}=\sin (\theta)
$$

and

$$
\theta=\arcsin (\mu)=\arccos \left(\sqrt{1-\mu^{2}}\right)
$$

is the angle of incidence in radians of an incident plane wave. This is defined as the angle between the normal of the panel and the direction of travel of the incident infinite plane wave with wave number $k$ in the fluid medium. This incident plane wave produces a forced bending wave of wave number $k_{b}$ in the panel.

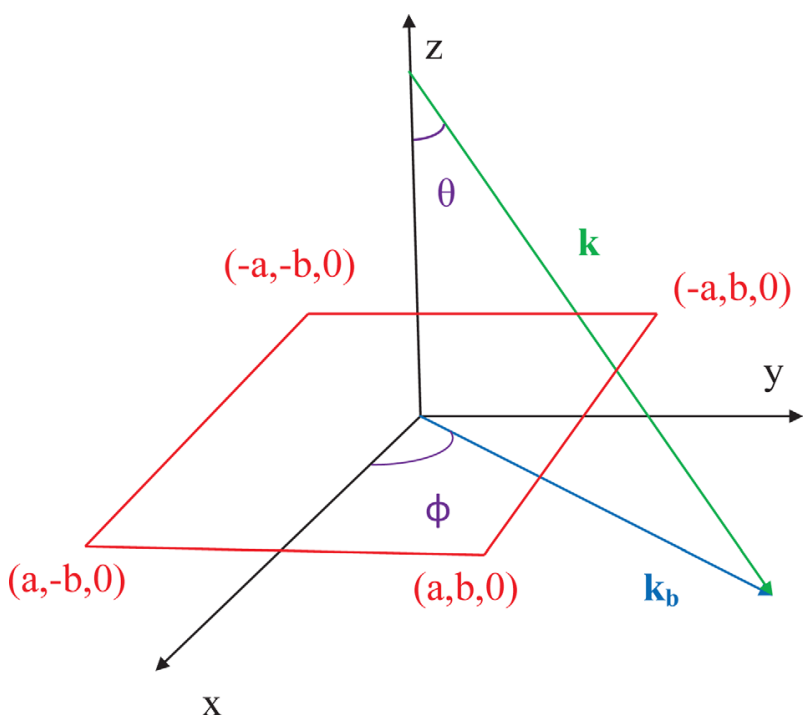

FIG. 1. (Color online) The geometry of the problem considered when the radiating surface $S$ is a rectangle. Note that if $\left|\mathbf{k}_{\mathbf{b}}\right|$ is greater than $|\mathbf{k}|, \theta$ does not exist as a real angle. 
The first line of Eq. (1) suggests for a bending wave, forced by an incident plane wave in the fluid medium, on a finite panel whose dimensions are large compared to the wavelength of sound in the fluid medium and which is mounted in an infinite baffle, that the real part of the average normalized specific wave impedance will be approximately $1 / \cos (\theta)$ and that the imaginary part will be close to zero, except for values of the incident angle which are close to grazing incidence $\left(\pi / 2\right.$ radians or $\left.90^{\circ}\right)$. This suggestion is correct.

The third line of Eq. (1) correctly suggests that the real part of the normalized specific radiation wave impedance of a freely propagating bending wave on a finite panel below the critical frequency of the panel in the fluid medium is close to zero and that the imaginary part is a mass like loading.

The normalized specific radiation impedance of a uniformly sinusoidally vibrating sphere of radius $r$ is (Cremer et al., 2005)

$$
z=\frac{(k r)^{2}}{1+(k r)^{2}}+j \frac{k r}{1+(k r)^{2}} .
$$

By symmetry, Eq. (4) also applies for a uniformly sinusoidally vibrating hemisphere of radius $r$ whose base is on an infinite rigid baffle. The real part of Eq. (4) also applies to panels or openings which are small compared to the wavelength of sound, are mounted in an infinite baffle and are vibrating uniformly (the angle of incidence of the forcing wave in the fluid medium $\theta$ is zero) if the area of the hemisphere is equal to the area of the panel or opening. Applying the same approach to the imaginary part produces the correct qualitative behavior, but the constant in the equation derived from Eq. (4) by applying this method needs to be modified to produce the correct quantitative behavior.

The first line of Eq. (1) and the real part of Eq. (4) also correctly suggest that the average normalized specific acoustic wave impedance for a uniformly sinusoidally vibrating panel or opening mounted in an infinite baffle tends to 1 as $k e$ tends to infinity. The uniform vibration means that $k_{b}=0, \mu=0$, and $\theta=0$.

Equations (1)-(4) give a semi-quantitative understanding of the average specific radiation wave impedance of a finite size rectangular panel mounted in an infinite rigid baffle. The aim of this paper is to develop and assess accurate approximations of the specific radiation wave impedance of a finite sized panel. These approximations will provide a more quantitative understanding of the specific radiation wave impedance.

\section{AVERAGE SPECIFIC RADIATION IMPEDANCE}

Consider a plane surface area $S$ whose area is also denoted by $S$, mounted in an infinite rigid plane baffle in the $x-y$ plane $z=0$, in which a two dimensional plane transverse velocity wave is propagating. The transverse velocity of the wave in the positive $z$ axis direction is

$$
u\left(\mathbf{r}_{0}\right)=u_{0} \exp \left(-j \mathbf{k}_{b} \cdot \mathbf{r}_{0}\right),
$$

where $\mathbf{r}_{0}=\left(x_{0}, y_{0}, z_{0}\right)$ is the position on the surface $S, \mathbf{k}_{b}$ $=\left(k_{x}, k_{y}, 0\right)$ is the wave number vector of the wave and $u_{0}$ is the complex amplitude of the wave. The sound pressure in the fluid medium on the positive $z$ side of the baffle at position $\mathbf{r}=(x, y, z)$ is given by the Rayleigh integral [see Fahy, 1985, Eq. (2.4)]

$$
p(\mathbf{r})=j k Z_{c} \iint_{S} g_{\omega}\left(\mathbf{r}, \mathbf{r}_{0}\right) u\left(\mathbf{r}_{0}\right) d \mathbf{r}_{0},
$$

where $g_{\omega}$ is the Green's function for a point source on an infinite rigid baffle which is given by

$$
g_{\omega}\left(\mathbf{r}, \mathbf{r}_{0}\right)=\frac{\exp (-j k R)}{2 \pi R},
$$

where

$$
R=\left|\mathbf{r}-\mathbf{r}_{0}\right|=\sqrt{\left(x-x_{0}\right)^{2}+\left(y-y_{0}\right)^{2}+\left(z-z_{0}\right)^{2}}
$$

and $k$ is the wave number in the fluid medium on the positive $\mathrm{z}$ side of the baffle.

The normalized specific acoustic wave impedance at $\mathbf{r}$ on the surface $S$ is

$$
z(\mathbf{r})=\frac{p(\mathbf{r})}{Z_{c} u(\mathbf{r})}=j k \iint_{S} g_{\omega}\left(\mathbf{r}, \mathbf{r}_{0}\right) \exp \left[j \mathbf{k}_{b} \cdot\left(\mathbf{r}-\mathbf{r}_{0}\right)\right] d \mathbf{r}_{0} .
$$

The average normalized specific acoustic wave impedance across the surface area $S$ is

$$
z=\frac{j k}{S} \iiint_{S} \int_{S} g_{\omega}\left(\mathbf{r}, \mathbf{r}_{0}\right) \exp \left[j \mathbf{k}_{b} \cdot\left(\mathbf{r}-\mathbf{r}_{0}\right)\right] d \mathbf{r} d \mathbf{r}_{0} .
$$

There are two main ways of reducing this quadruple integral to a double integral when $S$ is the rectangle given by

$$
|x| \leq a, \quad|y| \leq b, \quad z=0 .
$$

Note that $a$ and $b$ are half the lengths of the sides of the rectangle while many authors use them as the full lengths of the sides of the rectangle. Similarly the $e$ in this paper [see Eq. (41)] is half of the $e$ of Thomasson (1982). The first method for undertaking this reduction is presented in Appendix A of Li and Gibeling (2000) and Appendix 12.A of Allard and Atalla (2009). As Eq. (10) only depends on the difference $\mathbf{r}-\mathbf{r}_{0}$, it can be reduced to Eq. (64) of Brunskog (2012) and Eq. (12.A.11) of Allard and Atalla (2009) as shown in the following:

$$
\begin{aligned}
z= & \frac{j k}{2 \pi a b} \int_{0}^{2 a} \int_{0}^{2 b} \cos \left(k_{x} \kappa\right) \cos \left(k_{y} \tau\right) \frac{e^{-j k \sqrt{\kappa^{2}+\tau^{2}}}}{\sqrt{\kappa^{2}+\tau^{2}}} \\
& \times(2 a-\kappa)(2 b-\tau) d \kappa d \tau,
\end{aligned}
$$

where $\kappa=x-x^{\prime}$ and $\tau=y-y^{\prime}$ are the global co-ordinate transformations used to reduce the integral. Note that the $r^{2}$ 
from Brunskog [2012, Eqs. (63) and (64)] should be $\tau^{2}$ and the $k$ in the third line above Brunskog's Eq. (63) should be $\kappa$. Note that the function $F_{n}\left(u, u^{\prime}\right)$ from Allard and Atalla (2009) is only correct when the forcing plane wave is normally incident. In their notation, it should read as follows:

$$
F_{n}\left(u, u^{\prime}\right)=\cos \left[\frac{k_{t} L_{x} \cos (\varphi)}{2} u\right] \cos \left[\frac{k_{t} L_{x} \sin (\varphi)}{2} \frac{u^{\prime}}{r}\right] .
$$

In the function $K\left(u, u^{\prime}\right)$ in their Eq. (12.A.8), the argument of the exponential function should have a minus sign in front of it. The lower limit of the last integral in their Eq. (12.A.7) and the last integral on the left hand side of their Eq. (12.A.9) should be $2-u$ rather than $-u$.

The real part of Eq. (12) is

$$
\begin{aligned}
\operatorname{Re}(z)= & \frac{k^{2}}{2 \pi a b} \int_{0}^{2 a} \int_{0}^{2 b} \cos \left(k_{x} \kappa\right) \cos \left(k_{y} \tau\right) \\
& \times \operatorname{sinc}\left(k \sqrt{\kappa^{2}+\tau^{2}}\right)(2 a-\kappa)(2 b-\tau) d \kappa d \tau,
\end{aligned}
$$

where

$$
\operatorname{sinc}(x)=\frac{\sin (x)}{x}
$$

It should be noted that this is different from the definition of the sinc function in MATLAB. MATLAB defines its sinc function as $\operatorname{sinc}(\pi x)$ in terms of the sinc function defined in Eq. (15). The imaginary part of Eq. (12) is

$$
\begin{aligned}
\operatorname{Im}(z)= & \frac{k}{2 \pi a b} \int_{0}^{2 a} \int_{0}^{2 b} \cos \left(k_{x} \kappa\right) \cos \left(k_{y} \tau\right) \frac{\cos \left(k \sqrt{\kappa^{2}+\tau^{2}}\right)}{\sqrt{\kappa^{2}+\tau^{2}}} \\
& \times(2 a-\kappa)(2 b-\tau) d \kappa d \tau,
\end{aligned}
$$

which has a singularity at $\kappa=\tau=0$. It was found that MATLAB's adaptive integration routines cope reasonably well with the one point singularity.

The other way of reducing the quadruple integral is to express the Green's function in terms of its Fourier transform (Thomasson, 1982). According to Eq. (7.3.14) of Morse and Ingard (1968), the Green's function of a point source on an infinite rigid plane baffle can be written as

$$
g_{\omega}\left(\mathbf{r}, \mathbf{r}_{0}\right)=\frac{2}{(2 \pi)^{3}} \iiint \frac{\exp \left[j \mathbf{K} \cdot\left(\mathbf{r}_{0}-\mathbf{r}\right)\right]}{K^{2}-k^{2}} d \mathbf{K},
$$

where the factor of 2 in front of the triple integral has been included because the point sound source is on the infinite rigid plane baffle. $\mathbf{K}$ is defined as

$$
\mathbf{K}=\left(K_{x}, K_{y}, K_{z}\right)
$$

and

$$
K^{2}=|\mathbf{K}|^{2}=K_{x}^{2}+K_{y}^{2}+K_{z}^{2} .
$$

Using Eq. (17) in Eq. (10) and integrating with respect to $K_{z}, x, x_{0}, y$ and $y_{0}$ inside the two remaining integrals gives (Thomasson, 1982)

$$
\begin{aligned}
z= & \frac{k a}{\pi} \frac{k b}{\pi} \\
& \times \int_{-\infty}^{\infty} \int_{-\infty}^{\infty} \frac{\operatorname{sinc}^{2}\left[a\left(k_{x}-k \alpha_{x}\right)\right] \operatorname{sinc}^{2}\left[b\left(k_{y}-k \alpha_{y}\right)\right]}{\sqrt{1-\alpha_{x}^{2}-\alpha_{y}^{2}}} d \alpha_{x} d \alpha_{y} .
\end{aligned}
$$

It should be noted that the correct sign of the square root in Eq. (20) has to be chosen in order to obtain the correct sign for real and imaginary parts of the impedance. This choice of sign may be different on the two sides of the singularity which occurs on the unit circle $\alpha_{x}^{2}+\alpha_{y}^{2}=1$.

The real part of the impedance is obtained by calculating the integral in Eq. (20) over the unit disk $\alpha_{x}^{2}+\alpha_{y}^{2}<1$. Using circular coordinates and expressing the radius as a sine function removes the singularity on the unit circle $\alpha_{x}^{2}+\alpha_{y}^{2}=1$. Following Thomasson (1982), the following substitution is made:

$$
\left(\alpha_{x}, \alpha_{y}\right)=\left(\cos \left[\phi^{\prime}\right] \sin \left[\theta^{\prime}\right], \quad \sin \left[\phi^{\prime}\right] \sin \left[\theta^{\prime}\right]\right) .
$$

This gives

$$
\begin{aligned}
\operatorname{Re}(z)= & \frac{k a}{\pi} \frac{k b}{\pi} \int_{\phi^{\prime}=0}^{\phi^{\prime}=2 \pi} \int_{\theta^{\prime}=0}^{\theta^{\prime}=\pi / 2} \operatorname{sinc}^{2}\left\{a\left(k_{x}-k \sin \left[\theta^{\prime}\right] \cos \left[\phi^{\prime}\right]\right)\right\} \\
& \times \operatorname{sinc}^{2}\left\{b\left(k_{y}-k \sin \left[\theta^{\prime}\right] \sin \left[\phi^{\prime}\right]\right)\right\} \sin \left[\theta^{\prime}\right] d \theta^{\prime} d \phi^{\prime} .
\end{aligned}
$$

Because the real part of the average normalized specific acoustic wave impedance is the radiation efficiency, Eq. (22) can also be derived by calculating the acoustic power radiated by the rectangle across a very large hemisphere centered on the center of the rectangle and in conjunction with the infinite rigid baffle containing the rectangle enclosing the side of the rectangle whose radiation is being calculated (Sato, 1973).

The imaginary part of the impedance is obtained by calculating the integral in Eq. (20) over the area $\alpha_{x}^{2}+\alpha_{y}^{2}>1$ outside of the unit disk. The radius can be expressed as the inverse of a sine function in order to change the infinite radial limit to a finite limit. It should it noted that this is not absolutely essential as some of the MATLAB adaptive integration routines can use infinite limits, but it does simplify the calculations in мАтLAB. The following substitution is made:

$$
\left(\alpha_{x}, \alpha_{y}\right)=\left(\cos \left[\phi^{\prime}\right] / \sin \left[\theta^{\prime}\right], \quad \sin \left[\phi^{\prime}\right] / \sin \left[\theta^{\prime}\right]\right) .
$$

This gives

$$
\begin{aligned}
\operatorname{Im}(z)= & \frac{k a}{\pi} \frac{k b}{\pi} \int_{\phi^{\prime}=0}^{\phi^{\prime}=2 \pi} \int_{\theta^{\prime}=0}^{\theta^{\prime}=\pi / 2}\left(\operatorname{sinc}^{2}\left\{a\left(k_{x}-k \cos \left[\phi^{\prime}\right] / \sin \left[\theta^{\prime}\right]\right)\right\}\right. \\
& \left.\times \operatorname{sinc}^{2}\left\{b\left(k_{y}-k \sin \left[\phi^{\prime}\right] / \sin \left[\theta^{\prime}\right]\right)\right\} / \sin ^{2}\left[\theta^{\prime}\right]\right) d \theta^{\prime} d \phi^{\prime}
\end{aligned}
$$

The singularity at $\theta^{\prime}=0$ for all values of $\phi^{\prime}$ means that MATLAB's adaptive integration routines do struggle. It is 
often necessary to increase the maximum number of iterations or decrease the precision. As observed by Brunskog (2012), Eq. (12) is much more efficient numerically than Eqs. (22) and (24).

In order to calculate the total average specific wave impedance, the wave impedance must be integrated across all azimuthal angles and all angles of incidence. To allow this calculation to be performed first let $k_{b}(\phi)$ be the wave number of the plane transverse velocity wave which is propagating on the rectangle $S$ at the azimuthal angle $\phi$ to the $x$ axis. Then

$$
k_{b}(\phi)=\left|\mathbf{k}_{b}\right|=\sqrt{k_{x}^{2}+k_{y}^{2}}
$$

and

$$
k_{x}=k_{b}(\phi) \cos (\phi) \quad \text { and } \quad k_{y}=k_{b}(\phi) \sin (\phi),
$$

where $k_{b}(\phi)$ has been shown as a function of the azimuthal angle $\phi$ because it will in some cases depend on the direction of propagation, as is the case for a freely propagating wave on an orthotropic panel.

The weighted average of the impedance over azimuthal angle with weighting function $w(\phi)$ is

$$
z_{a z}=\int_{0}^{2 \pi} w(\phi) z(\phi) d \phi / \int_{0}^{2 \pi} w(\phi) d \phi .
$$

If $w(\phi)$ and $k_{b}(\phi)$ are symmetrical functions about the $x$ and $y$ axes, the ranges of integration over the azimuthal angle can be reduced to 0 to $\pi / 2$ radians by symmetry. If $w(\phi)$ and $k_{b}(\phi)$ are constant functions of azimuthal angle and the rectangle $S$ is a square, the ranges of integration over the azimuthal angle can be reduced to 0 to $\pi / 4$ radians by symmetry. The weighting function $w(\phi)$ can be used to account for the fact that the wave impedance of an orthotropic panel varies with the azimuthal angle of propagation.

If the transverse velocity wave is forced by a plane sound wave incident from either side of the panel with an incidence angle of $\theta$ to the normal of the surface $S$ and an azimuthal angle of $\phi$ to the $x$ axis, then

$$
k_{b}(\phi)=k \sin (\theta)
$$

is constant as a function of the azimuthal angle $\phi$, and

$$
k_{x}=k \sin (\theta) \cos (\phi) \quad \text { and } \quad k_{y}=k \sin (\theta) \sin (\phi) .
$$

Figures 2 and 3 show the numerically calculated real part and imaginary part, respectively, of the normalized surface averaged and azimuthally averaged specific radiation wave impedance as a function of the ratio $\mu$ of the bending wave number $k_{b}$ of a square panel of side length $2 e$, mounted in an infinite rigid baffle, to the wave number $k$ of sound in the medium into which the panel is radiating. The legend shows the value of $k e$.

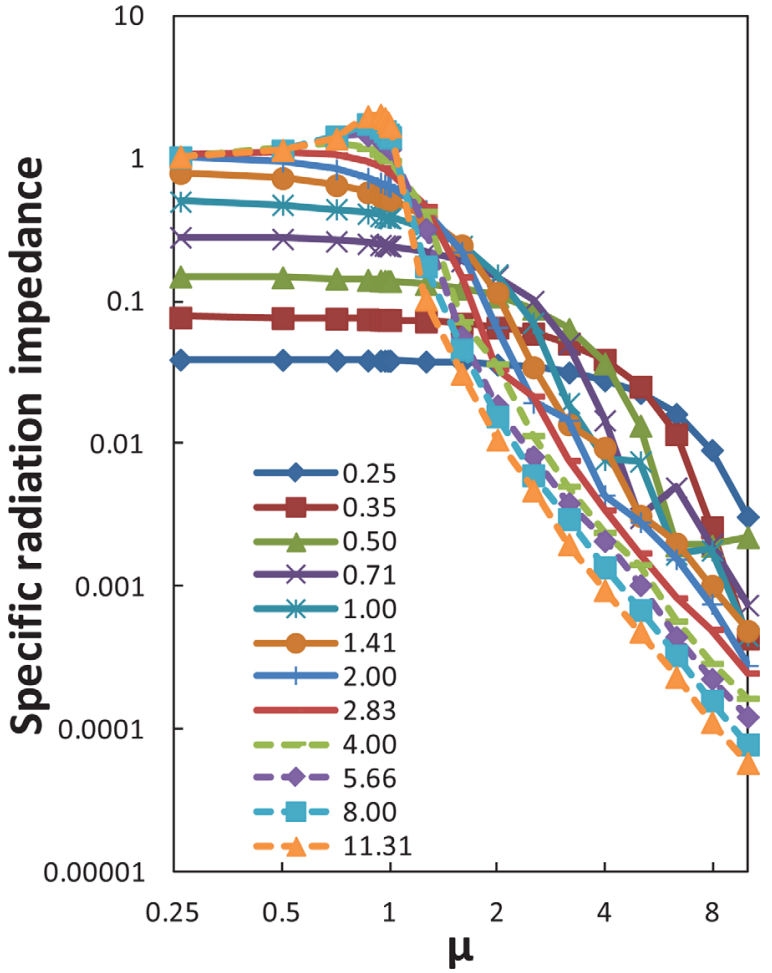

FIG. 2. (Color online) The numerically calculated real part of the normalized surface averaged and azimuthally averaged specific radiation impedance as a function of the ratio $\mu$ of the bending wave number $k_{b}$ of a square panel, of side length $2 e$ mounted in an infinite rigid baffle, to the wave number $k$ of sound in the medium into which the panel is radiating. The legend shows the value of $k e$.

The incident diffuse sound field forced radiation impedance is the average of $z$ over all solid angles of incidence as shown in the following:

$$
\langle z\rangle=\int_{0}^{\pi / 2} z_{a v} \sin (\theta) d \theta .
$$

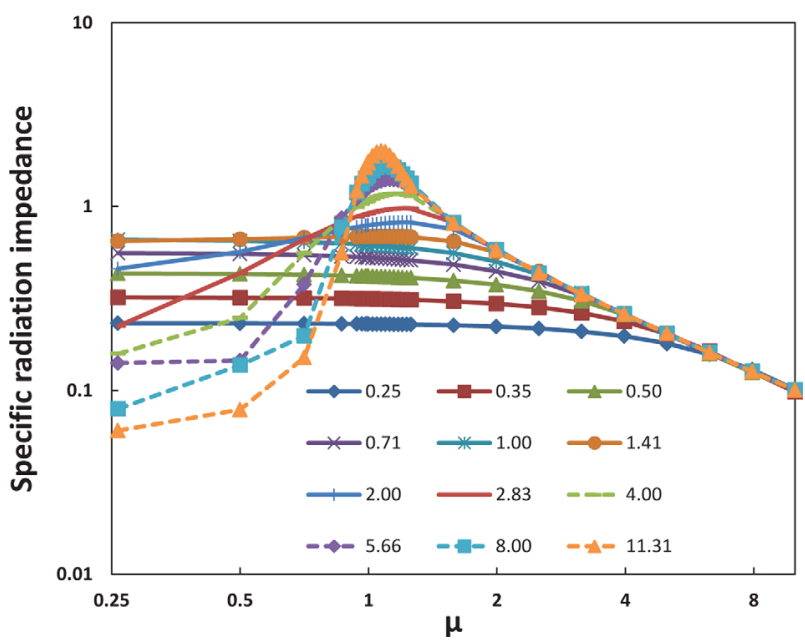

FIG. 3. (Color online) The numerically calculated imaginary part of the normalized surface averaged and azimuthally averaged specific radiation impedance as a function of the ratio $\mu$ of the bending wave number $k_{b}$ of a square panel, of side length $2 \mathrm{e}$ mounted in an infinite rigid baffle, to the wave number $k$ of sound in the medium into which the panel is radiating. The legend shows the value of $k e$. 


\section{NUMERICAL ACCURACY}

Thomasson (1982) used Eqs. (22) and (24) to tabulate, to nearest 0.01 , the real and imaginary parts of the azimuthally averaged impedance of a square panel. The tabulated data is presented for values of $k e$ in half octave steps from 0.25 to 64 and values of $\theta$ in $15^{\circ}$ increments from 0 to $90^{\circ}$ in his Table I. To obtain the azimuthal average, Thomasson replaced Eq. (27) where $w(\phi)$ is equal to one with an average of the values at $\phi$ equal to $0^{\circ}, 15^{\circ}, 30^{\circ}, 45^{\circ}$. For $k e=32$, values at $\theta$ equals $70^{\circ}, 80^{\circ}$, and $85^{\circ}$ and for $k e=64$, values at $\theta$ equals $80^{\circ}$ and $85^{\circ}$ were read from Thomasson's Figure B3. MATLAB's adaptive integral functions were used to evaluate Eqs. (12) and (27) with $w(\phi)$ equal to 1 for comparison with Thomasson's results. MATLAB's default settings were used in all cases. The real results tabulated by Thomasson were greater than the MATLAB results by between 0.0067 and -0.0181 . The mean and standard deviation of the differences were 0.0001 and 0.036. If the results read from Thomasson's graph were removed, the lower limit became -0.0049 . Thomasson's imaginary results were greater than the MATLAB results by between 0.0147 and -0.0807 . The mean and standard deviation of the differences were -0.0008 and 0.0082 . If the results read from Thomasson's graph were removed, the lower limit became -0.0056 . Given that Thomasson's results were rounded to the nearest 0.01 , this was a satisfactory result. The differences in excess of Thomasson's maximum rounding error of 0.005 were probably due to his averaging of the results for four azimuthal angles rather than integrating over azimuthal angle.

Sato (1973) used Eq. (22) to tabulate the real part of the azimuthally averaged impedance of a square to the nearest $0.1 \mathrm{~dB}$ for values of $k e$ of $0.5,0.75,1,1.5,2,3,4,6,8,12$, $16,24,32,48$ and 64 and values of $\theta$ in $15^{\circ}$ increments from 0 to $90^{\circ}$ in his Table I. He also graphed results for values of $k e$ in octave steps from 2 to 64 and values of $\theta$ in $5^{\circ}$ increments from 0 to $90^{\circ}$ in his Fig. 4. Equations (12) and (27) with $w(\phi)$ equal to 1 were evaluated with the adaptive integral functions of MATLAB using their default settings and compared with Sato's results. Sato's real results were greater than the MATLAB results by between 0.14 and $-0.24 \mathrm{~dB}$. The mean and standard deviation of the differences were 0.03 and $0.07 \mathrm{~dB}$. If the results read from Sato's graph were removed, the lower limit became $-0.15 \mathrm{~dB}$. Given that Sato's results were rounded to the nearest $0.1 \mathrm{~dB}$, this was a reasonable result.

Sato (1973) used Eqs. (22) and (30) to tabulate the real part of the incident diffuse sound field forced radiation impedance to the nearest $0.01 \mathrm{~dB}$ for values of $k e$ of $0.5,0.75$, $1,1.5,2,3,4,6,8,12,16,24,32,48$, and 64 in his Table I. Equations (12) and (27) with $w(\phi)$ equal to 1, and Eq. (30) were evaluated with the adaptive integral functions of MATLAB using their default settings and compared with Sato's results. Sato's real results were greater than the MATLAB results by between 0.050 and $-0.081 \mathrm{~dB}$. The mean and standard deviation of the differences were -0.029 and $0.39 \mathrm{~dB}$. While these differences are significantly bigger than Sato's maximum rounding error of $0.005 \mathrm{~dB}$, they are too small to be of any practical significance. It should be noted that it took nearly four hours to calculate the real and imaginary parts of the impedance for the 15 values of $k e$. This is because four nested integrals need to be evaluated. The results for the two smallest values of ke only took about one and half minutes to calculate, but the time required for a solution to be calculated increased as ke increased. This shows the importance of having approximations for the impedance.

A comparison was made between the numerical results of Sato (1973) and Thomasson (1982) for the real part of the impedance across those values of $k e$ and incidence excitation angle $\theta$ for which they had both calculated results. The results from Sato (1973) were greater than those from Thomasson (1982) by between 0.145 and $-0.234 \mathrm{~dB}$. The mean and standard deviation of the differences were 0.019 and $0.068 \mathrm{~dB}$. If the values that had to be read from graphs were removed, the lower limit became $-0.131 \mathrm{~dB}$ and the upper limit remained unchanged.

Stenzel (1952) tabulated to five decimal places the real and imaginary parts of the impedance of rectangles with side length ratios of $b / a$ equal to $0.1,0.2,0.5$, and 1 for the normally incident excited case $\left(\theta=0, k_{b}=0\right.$, or $\left.\mu=0\right)$. The calculations were made for values of $k a$ from 0.5 to 5 in steps of 0.5 . For the real part, the calculations were also made for $k a$ equal to 6 . Stenzel's real results were greater than the MATLAB results by between 0.00084 and -0.00318 . The mean and standard deviation of the differences were 0.00006 and 0.00050. Stenzel's imaginary results were greater than the MATLAB results by between 0.001916 and -0.00081 . The mean and standard deviation of the differences were 0.00051 and 0.00304 .

\section{APPROXIMATIONS}

If $k_{b} R \ll 1$ and $k R \ll 1$ then the exponential function in Eq. (9) is approximately 1 and the imaginary part of the Green's function in Eq. (9) is approximately $-j k /(2 \pi)$. Making these approximations in Eq. (9) and performing the integrals gives

$$
\begin{aligned}
\operatorname{Re}(z)=\frac{k^{2} S}{2 \pi} & =\frac{2 k^{2} a b}{\pi} \\
& \text { if } k a \ll 1, \quad k b \ll 1, k_{b} a \ll 1, k_{b} b \ll 1 .
\end{aligned}
$$

It should be noted that Eq. (31) does not depend on the angle of incidence, azimuthal angle or the shape of the surface $S$ or if $S$ is a rectangle on the ratio $b / a$.

If $k a \gg 1$ and $k b \gg 1$ then the product of the sinc squared functions in Eq. (20) has a sharp maximum at the point $\left(\alpha_{x}, \alpha_{y}\right)=\left(k_{x} / k, k_{y} / k\right)$. Providing that the location of this maximum is not too close to the singularity on the unit circle $\alpha_{x}^{2}+\alpha_{y}^{2}=1$, the integrals in Eq. (20) can be approximated by setting the square root to its value at this location. The integrals can then be evaluated by using integral number 3.821.9 of Gradshteyn and Ryzhik (1965). This gives 


$$
z= \begin{cases}\frac{1}{\sqrt{1-\frac{k_{x}^{2}}{k^{2}}-\frac{k_{y}^{2}}{k^{2}}}}=\frac{1}{\sqrt{1-\frac{k_{b}^{2}(\phi)}{k^{2}}}}=\frac{1}{\sqrt{1-\mu^{2}}} & \text { if } k_{b}^{2}(\phi)=k_{x}^{2}+k_{y}^{2}<k^{2} \\ \frac{j}{\sqrt{\frac{k_{x}^{2}}{k^{2}}+\frac{k_{y}^{2}}{k^{2}}-1}}=\frac{j}{\sqrt{\frac{k_{b}^{2}(\phi)}{k^{2}}-1}}=\frac{j}{\sqrt{\mu^{2}-1}} & \text { if } k_{b}^{2}(\phi)=k_{x}^{2}+k_{y}^{2}>k^{2} .\end{cases}
$$

The first line of Eq. (32) agrees with Eq. (7.6) of Leppington et al. (1982). Leppington et al. (1982) have also shown that the first line of Eq. (32) requires $k \min (a, b)(1-\mu) \gg 1$. Eq. (32) does not depend on $a$ or $b$, and if as will often be the case $k_{b}(\phi)$ is constant as a function of $\phi$, it does not depend on the azimuthal angle $\phi$. Note as indicated above, the correct sign of the square root needs to be chosen. If Eq. (29) applies then Eq. (32) becomes

$$
z=\frac{1}{\cos (\theta)} \quad \text { if } \theta<\frac{\pi}{2}
$$

A pair of equations are presented that govern how close $\theta$ can be to $\pi / 2$, while still retaining sufficient accuracy. According to Davy (2009), Eq. (33) and hence the first line Eq. (32) are approximately correct if

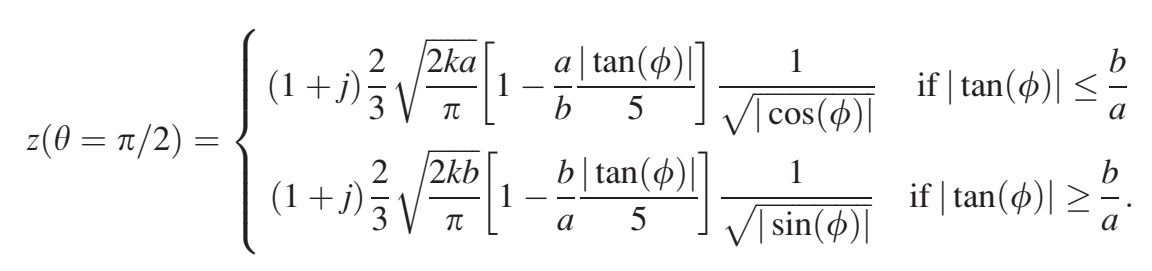

The real part of Eq. (36) is the same as Eq. (6.25) of Leppington et al. (1982). Notice that Eq. (36), unlike Eqs. (31), (32), and (33), does depend on the azimuthal angle $\phi$ and the ratio of the length of the sides of the rectangle $b / a$. According to Eqs. (7.9) and (7.10) of Leppington et al. (1982), the azimuthal average of Eq. (36) is

$$
z_{a v}(\theta=\pi / 2)=(1+j) \sqrt{k \min (a, b)} L\left(\frac{\min (a, b)}{\max (a, b)}\right),
$$

where

$$
L(x)=\frac{4}{15 \pi} \sqrt{\frac{x}{\pi}} \int_{0}^{1}(5-t)\left\{\left(t^{2}+x^{2}\right)^{3 / 4}+\left(1+x^{2} t^{2}\right)^{-3 / 4}\right\} d t .
$$

According to Leppington et al. (1982), over the range $1 / 5 \leq x \leq 1$, the following approximation has an error of less than $4 \%$ :

$$
\begin{aligned}
\sqrt{1-\frac{k_{x}^{2}}{k^{2}}-\frac{k_{y}^{2}}{k^{2}}} & =\sqrt{1-\frac{k_{b}^{2}(\phi)}{k^{2}}}=\sqrt{1-\mu^{2}} \\
& =\cos (\theta) \geq \min \left(g \sqrt{\frac{\pi}{2 k e}}, 1\right),
\end{aligned}
$$

where $g$ equals 1.3. This suggests that the second line of Eq. (32) is approximately correct if

$$
\sqrt{\frac{k_{x}^{2}}{k^{2}}+\frac{k_{y}^{2}}{k^{2}}-1}=\sqrt{\frac{k_{b}^{2}(\phi)}{k^{2}}-1}=\sqrt{\mu^{2}-1} \geq h \sqrt{\frac{\pi}{2 k e}} .
$$

Numerical evaluation shows that $h$ should be set to 1.7 for the real part of the second line of Eq. (32) and to 1.6 for the imaginary part of the second line of Eq. (32) when using Eq. (35).

If $k a \gg 1, k b \gg 1, \theta=\pi / 2$ and Eq. (29) applies, Thomasson (1982) has shown that approximately

$$
L(x)=0.5-0.15 x \text {. }
$$

Thomasson (1982) has shown that if $1 / 4<b / a<4$, the azimuthal average of Eq. (36) can be approximated to within $3 \%$ by

$$
z_{a v}(\theta=\pi / 2)=0.9616(1+j) \frac{2}{3} \sqrt{\frac{2 k e}{\pi}},
$$

where

$$
2 e=4 S / U=4 a b /(a+b) .
$$

$S$ is the area of the rectangle and $U$ is the perimeter of the rectangle. This compares well with Eq. (27) of Davy (2009), which is

$$
\operatorname{Re}\left[z_{a v}(\theta=\pi / 2)\right]=\frac{2}{3} \sqrt{\frac{2 k e}{\pi}}-0.124
$$


Thomasson (1982) gave approximations for $k e \leq 0.25$ and for $k e \geq 64$. For $0.25 \leq k e \leq 64$ he gave numerically calculated values for a square at half octave intervals. The approximations from Thomasson (1982) are as follows:

$$
\begin{aligned}
z_{a v}= & 1 / \sqrt{\cos ^{2}(\theta)-j 2 \beta \sin (\theta) / k e+[\beta /(k e)]^{2}} \\
& \text { if } k e \geq 64 \quad \text { where } \beta=0.956 \\
z_{a v}= & 2 k^{2} a b / \pi+j 2 k[b H(a / b)+a H(b / a)] / \pi \\
& \text { if } k e \leq 0.25,
\end{aligned}
$$

where

$$
H(q)=\ln \left(\sqrt{1+q^{2}}+q\right)-\left(\sqrt{1+q^{2}}-1\right) /(3 q) .
$$

There are typographical errors in Brunskog [2012, Eqs. (65) and (67)], which are his versions of version of Eqs. (43) and (45). The square root sign in the denominator of Brunskog's Eq. (65) should include all terms in the denominator and the last $+q$ in Brunskog's Eq. (67) should be -1 . For a square panel Eq. (44) becomes

$$
z_{a v}=2(k e)^{2} / \pi+0.946 j k e \text { if } k e \leq 0.25 .
$$

Equating the area of the square to the area of the hemisphere, $4 e^{2}=2 \pi r^{2}$ and $r=e \sqrt{2 / \pi}$. Putting these values into Eq. (4) and assuming that $(k r)^{2} \ll 1$ gives

$$
\begin{aligned}
z_{a v} & =2(k e)^{2} / \pi+\sqrt{2 / \pi} j k e \\
& =2(k e)^{2} / \pi+0.798 j k e \quad \text { if }(k r)^{2} \ll 1 .
\end{aligned}
$$

This approach gives the correct real part of Eq. (46), but the constant in the imaginary part is slightly in error. The real parts of Eqs. (44), (46), and (47) also agree with Eq. (31). For $k e \gg 1$, Eq. (43) shows the $1 / \cos (\theta)$ behavior predicted by Eq. (1). For $\theta=\pi / 2$ radians $\left(90^{\circ}\right)$ and $(k e)^{2} \gg 1$, the real and imaginary parts of Eq. (43) are both positive and approximately equal. For $\theta$ not near $\pi / 2$ radians $\left(90^{\circ}\right)$ and $k e \gg 1$, the imaginary part of Eq. (43) is very much less than the real part.

The second line of Eq. (32) indicates that the real part of $z$ is approximately zero if $k_{b}^{2}(\phi)=k_{x}^{2}+k_{y}^{2}>k^{2}$. While the real part is small compared to the imaginary part, it is not actually zero. Leppington et al. (1982) have evaluated the azimuthally average normalized radiation impedance for the case of standing waves of the form

$$
u(x, y, 0)=u_{0} \sin \left[k_{x}(x+a)\right] \sin \left[k_{y}(y+b)\right]
$$

rather than for the traveling waves described by Eq. (5). The standing wave of Eq. (48) can be expressed as the product of the sum of two waves traveling in opposite directions parallel to the $x$ axis with the sum of two waves traveling in opposite directions parallel to the $y$ axis. This formulation means that the integrand whose integral is approximated by Leppington et al. (1982) is now the sum of four terms rather than just the single term of the integrand in this paper. The phase of the four traveling waves, which is controlled by the boundary conditions of the panel, is known to influence the real part of the impedance when $\mu$ is greater than one. Because of the extra terms in the integrand, Eq. (7.7) of Leppington et al. (1982) is used without its natural logarithm term,

$$
\begin{aligned}
\operatorname{Re}\left(z_{a v}\right)= & \frac{2}{\pi k e\left(\mu^{2}-1\right)^{3 / 2}} \\
& \text { if } \mu>1 \text { and } k \min (a, b)(\mu-1) \gg 1,
\end{aligned}
$$

where

$$
\mu=\frac{k_{b}}{k}=\frac{\sqrt{k_{x}^{2}+k_{y}^{2}}}{k} .
$$

The appearance of $e$ in Eq. (49) as well as in Eq. (40) shows the importance of $e$ as a measure of the size of a rectangular panel.

\section{COMBINED FORMULAS}

Davy (2009) combined high and low frequency approximations for the real part of the averaged normalized specific forced radiation wave impedance which is also equal to the radiation efficiency. The aim of this paper is to combine the low and high frequency approximations from Thomasson (1982) in order to cover the whole frequency range. This would give a formula for the imaginary part which is not provided by Davy (2009).

The case when $\mu$ is less than or equal to 1 is considered first. Following Davy (2009), the low $x_{L}$ and $x_{H}$ approximations are combined using the following formula:

$$
x=\frac{1}{\sqrt[n]{\frac{1}{x_{L}^{n}}+\frac{1}{x_{H}^{n}}}} .
$$

The real part of the specific forced radiation wave impedance is given by the $x$ in Eq. (51) when $x_{L}$ and $x_{H}$ are the real parts of Eqs. (44) and (43), respectively, and $n=2$.

The imaginary part is more complicated. For a normally incident exciting wave $(\theta=0)$, Eq. (43) gives a zero imaginary part. Although it is small for large values of ke, the imaginary part is not completely zero. A straight line of best fit was applied in the log-log domain to the numerical calculations of Thomasson (1982) for the imaginary part for a normally incident exciting wave $(\theta=0)$ versus $k e$ for values of $k e$ from 1.41 to 64 . This produced the following equation:

$$
\operatorname{Im}[z(\theta=0)]=\frac{0.67}{k e} \quad \text { if } k e \geq 1.41 .
$$

Note that apart from the 0.67 scaling factor, this is in agreement with the high frequency asymptotic behavior of the imaginary part of Eq. (4). The imaginary part of the specific forced radiation wave impedance for a normally incident exciting wave $(\theta=0)$ for all values of $k e$ is obtained by 
using Eq. (51) with $n=3$. The low frequency component $x_{L}$ is the imaginary part of Eq. (44) and the high frequency component $x_{H}$ is given by Eq. (52). The value of the imaginary part for any angle of incidence is calculated as the maximum of the imaginary part for the $\theta=0$ case as described in this paragraph and the imaginary part of Eq. (43).

For the case when $\mu$ is greater than one, the radiation impedance was approximated by using the second line of Eq. (32) to calculate the imaginary part and Eq. (49) to calculate the real part when Eq. (35) is satisfied. If Eq. (35) is not satisfied, the radiation impedance was calculated by interpolating between $\mu$ equals one and the value of $\mu$ given by solving Eq. (35) with the equality sign. Note that the $\mu$ equals one case is the $\theta$ equals $\pi / 2$ radians or $90^{\circ}$ case.

\section{SUMMARY OF COMBINED APPROXIMATE CALCULATION METHOD}

From Eq. (2) calculate

$\mu=\frac{k_{b}}{k}=\sin (\theta)$

where the second equality only applies if $k_{b} \leq k$. From Eq. (41) calculate

$$
k e=\frac{2 k a b}{a+b} .
$$

If $\mu \leq 1$ calculate

$\kappa=\frac{0.956}{k e}$.

From Eq. (43) calculate

$$
z_{h}=z_{h r}+j z_{h i}=\frac{1}{\sqrt{1+(\kappa-j \mu)^{2}}} .
$$

From Eq. (44) calculate

$$
z_{l}=z_{l r}+j z_{l i}=\frac{2 k^{2} a b}{\pi}+j \frac{2 k}{\pi}\left[b H\left(\frac{a}{b}\right)+a H\left(\frac{b}{a}\right)\right],
$$

where from Eq. (45)

$$
H(q)=\ln \left(\sqrt{1+q^{2}}+q\right)-\frac{\sqrt{1+q^{2}}-1}{3 q} .
$$

Using Eq. (51) with $n=2$ calculate

$$
z_{r}=\frac{1}{\sqrt{\frac{1}{z_{l r}^{2}}+\frac{1}{z_{h r}^{2}}}} .
$$

From Eq. (52) calculate

$$
z_{h i 0}=\frac{0.67}{k e} .
$$

Using Eq. (51) with $n=3$ calculate

$$
z_{i 0}=\frac{1}{\sqrt[3]{\frac{1}{z_{l i}^{3}}+\frac{1}{z_{h i 0}^{3}}}} .
$$

Calculate

$$
z_{i}=\max \left(z_{i 0}, z_{h i}\right) .
$$

Calculate

$$
z=z_{r}+j z_{i}
$$

Else if $\mu>1$, set $h_{r}=1.7$ and $h_{i}=1.6$ and calculate $\mu_{x}$ where $x$ equals $r$ and $i$ using Eq. (35), as follows:

$$
\mu_{x}=\sqrt{1+\frac{\pi h_{x}^{2}}{2 k e}}
$$

where $x$ equals $r$ or $i$. If $\mu \geq \mu_{r}$, using Eq. (49) calculate

$$
z_{r}=\frac{2}{\pi k e\left(\mu^{2}-1\right)^{3 / 2}} .
$$

If $1<\mu<\mu_{r}$ calculate the real part $z_{m r}$ using Eq. (65) with $\mu=\mu_{r}$.

Calculate the real part $z_{1 r}$ as described for the $\mu \leq 1$ case with $\mu=1$.

Interpolate

$$
z_{r}=\frac{\left(\mu_{r}-\mu\right) z_{1 r}+(\mu-1) z_{m r}}{\mu_{r}-1} .
$$

If $\mu \geq \mu_{i}$, using Eq. (32) calculate

$z_{i}=\frac{1}{\sqrt{\mu^{2}-1}}$.

If $1<\mu<\mu_{i}$ calculate the imaginary part $z_{m i}$ using Eq. (67) with $\mu=\mu_{i}$.

Calculate the imaginary part $z_{1 i}$ as described for the $\mu \leq 1$ case with $\mu=1$.

Interpolate

$$
z_{i}=\frac{\left(\mu_{i}-\mu\right) z_{1 i}+(\mu-1) z_{m i}}{\mu_{i}-1} .
$$

If $\mu>1$, calculate

$$
z=z_{r}+j z_{i}
$$

where $z_{r}$ is given by Eq. (65) or Eq. (66) and $z_{i}$ is given by Eq. (67) or Eq. (68).

The area averaged and azimuthally averaged specific radiation wave impedance of a finite rectangular panel is given by Eq. (63) or Eq. (69). 
TABLE I. Difference in decibels between the combined approximate method developed in this paper and the numerical calculations of Thomasson (1982) for the real part of the specific forced radiation wave impedance of a square panel of side length $2 e$ when $\mu \leq 1$.

\begin{tabular}{|c|c|c|c|c|c|c|c|c|c|c|}
\hline & $\mu=0.000$ & $\mu=0.259$ & $\mu=0.500$ & $\mu=0.707$ & $\mu=0.866$ & $\mu=0.940$ & $\mu=0.966$ & $\mu=0.985$ & $\mu=0.996$ & $\mu=1.000$ \\
\hline ke & $0^{\circ}$ & $15^{\circ}$ & $30^{\circ}$ & $45^{\circ}$ & $60^{\circ}$ & $70^{\circ}$ & $75^{\circ}$ & $80^{\circ}$ & $85^{\circ}$ & $90^{\circ}$ \\
\hline 0.25 & -0.1 & -0.1 & -0.1 & -0.1 & -0.1 & & -0.1 & & & -0.1 \\
\hline 0.35 & -0.1 & -0.1 & -0.1 & -0.1 & -0.2 & & 0.4 & & & 0.4 \\
\hline 0.50 & 0.0 & 0.0 & 0.0 & 0.3 & 0.3 & & 0.2 & & & 0.2 \\
\hline 0.71 & 0.0 & 0.0 & 0.1 & 0.2 & 0.3 & & 0.3 & & & 0.5 \\
\hline 1.00 & -0.3 & -0.2 & -0.1 & 0.2 & 0.4 & & 0.5 & & & 0.5 \\
\hline 1.41 & -0.7 & -0.6 & -0.2 & 0.2 & 0.4 & & 0.6 & & & 0.6 \\
\hline 2.00 & -1.0 & -0.8 & -0.4 & 0.2 & 0.5 & & 0.7 & & & 0.7 \\
\hline 2.83 & -0.4 & -0.5 & -0.5 & 0.0 & 0.4 & & 0.5 & & & 0.5 \\
\hline 4.00 & 0.1 & -0.1 & -0.5 & -0.3 & 0.2 & & 0.4 & & & 0.4 \\
\hline 5.66 & -0.2 & -0.1 & -0.1 & -0.5 & -0.1 & & 0.3 & & & 0.3 \\
\hline 8.00 & -0.1 & 0.0 & 0.0 & -0.3 & -0.3 & & 0.2 & & & 0.2 \\
\hline 11.31 & -0.1 & 0.0 & 0.0 & 0.0 & -0.5 & & 0.1 & & & 0.2 \\
\hline 16.00 & 0.0 & 0.0 & 0.0 & -0.1 & -0.4 & & 0.0 & & & 0.1 \\
\hline 22.63 & 0.0 & 0.0 & 0.0 & 0.0 & -0.1 & & -0.2 & & & 0.1 \\
\hline 32.00 & 0.0 & 0.0 & 0.0 & 0.0 & -0.1 & -0.4 & -0.4 & 0.0 & 0.1 & 0.1 \\
\hline 45.25 & 0.0 & 0.0 & 0.0 & 0.0 & 0.0 & & -0.5 & & & 0.0 \\
\hline 64.00 & 0.0 & 0.0 & 0.0 & 0.0 & 0.0 & & -0.4 & -0.3 & 0.1 & 0.0 \\
\hline
\end{tabular}

\section{COMPARISON WITH NUMERICALLY CALCULATED VALUES}

Tables I and II show the amounts in decibels by which the real and imaginary parts of the combined approximate method developed in this paper for the forced radiation impedance were greater than the numerical calculations from Thomasson (1982) for the real and imaginary parts respectively of the specific forced radiation wave impedance of a square panel of side length $2 e$ when $\mu$ is less than or equal to one. For the real part, the differences are between 0.7 and $-1.0 \mathrm{~dB}$. The mean and standard deviation of the differences were 0.01 and $0.30 \mathrm{~dB}$. For the imaginary part, the differences are between 2.3 and $-2.6 \mathrm{~dB}$. The mean and standard deviation of the differences were -0.31 and $0.90 \mathrm{~dB}$. The extreme differences occur in region of ke equals 2. However the imaginary part also has a difference of $1.7 \mathrm{~dB}$ for $k e$ equals 64 at an angle of incidence of $60^{\circ}$. This difference occurs where the imaginary part is increasing very rapidly from a very low value for angles of incidence close to normal to a very large value at grazing angles of incidence.

Table III shows the amount in decibels that the method from Davy (2009) was greater than that of the Thomasson (1982) numerical calculations for the real part of the specific forced radiation wave impedance of a square panel of side length $2 e$ when $\mu$ is less than or equal to one. The differences are between 0.4 and $-0.6 \mathrm{~dB}$. The mean and standard deviation of the differences were -0.03 and $0.19 \mathrm{~dB}$. Thus, the method of Davy (2009) is in slightly better agreement with the numerical calculations of Thomasson (1982) for the real

TABLE II. Difference in decibels between the combined approximate method developed in this paper and the numerical calculations from Thomasson (1982) for the imaginary part of the specific forced radiation wave impedance of a square panel of side length $2 e$ when $\mu \leq 1$.

\begin{tabular}{|c|c|c|c|c|c|c|c|c|c|c|}
\hline & $\mu=0.000$ & $\mu=0.259$ & $\mu=0.500$ & $\mu=0.707$ & $\mu=0.866$ & $\mu=0.940$ & $\mu=0.966$ & $\mu=0.985$ & $\mu=0.996$ & $\mu=1.000$ \\
\hline ke & $0^{\circ}$ & $15^{\circ}$ & $30^{\circ}$ & $45^{\circ}$ & $60^{\circ}$ & $70^{\circ}$ & $75^{\circ}$ & $80^{\circ}$ & $85^{\circ}$ & $90^{\circ}$ \\
\hline 0.25 & 0.1 & 0.1 & 0.1 & 0.1 & 0.1 & & 0.1 & & & 0.1 \\
\hline 0.35 & 0.2 & 0.2 & 0.2 & 0.2 & 0.3 & & 0.3 & & & 0.3 \\
\hline 0.50 & 0.4 & 0.4 & 0.4 & 0.4 & 0.5 & & 0.5 & & & 0.5 \\
\hline 0.71 & 0.3 & 0.3 & 0.4 & 0.5 & 0.6 & & 0.6 & & & 0.6 \\
\hline 1.00 & -0.4 & -0.4 & -0.3 & -0.2 & -0.2 & & -0.1 & & & -0.1 \\
\hline 1.41 & -1.4 & -1.4 & -1.5 & -1.6 & -1.6 & & -1.6 & & & -1.5 \\
\hline 2.00 & -0.9 & -1.4 & -2.3 & -2.6 & -1.7 & & -1.1 & & & -1.0 \\
\hline 2.83 & 2.3 & 0.3 & -2.6 & -2.5 & -1.5 & & -0.9 & & & -0.7 \\
\hline 4.00 & -0.5 & 0.2 & -1.7 & -2.2 & -1.4 & & -0.7 & & & -0.5 \\
\hline 5.66 & 1.2 & -0.7 & -1.0 & -1.4 & -1.4 & & -0.6 & & & -0.4 \\
\hline 8.00 & -0.3 & 0.2 & -2.0 & 0.3 & -1.4 & & -0.6 & & & -0.2 \\
\hline 11.31 & -0.1 & -0.1 & -1.0 & 0.3 & -0.8 & & -0.6 & & & -0.2 \\
\hline 16.00 & 0.2 & 0.2 & -1.2 & -0.1 & 0.9 & & -0.7 & & & -0.1 \\
\hline 22.63 & -0.1 & -0.1 & -0.9 & 0.2 & 1.6 & & -0.9 & & & -0.1 \\
\hline 32.00 & 0.2 & 0.2 & -1.2 & 0.7 & 0.5 & 0.6 & -0.8 & -0.5 & -0.1 & -0.1 \\
\hline 45.25 & -1.3 & -1.3 & -0.9 & 0.2 & 1.2 & & -0.2 & & & 0.0 \\
\hline 64.00 & 0.2 & 0.2 & 0.6 & 0.0 & 1.7 & & 1.4 & -0.8 & -0.1 & 0.0 \\
\hline
\end{tabular}


TABLE III. Difference in decibels between the method of Davy (2009) and the numerical calculations of Thomasson (1982) for the real part of the specific forced radiation wave impedance of a square panel of side length $2 e$ when $\mu \leq 1$.

\begin{tabular}{|c|c|c|c|c|c|c|c|c|c|c|}
\hline & $\mu=0.000$ & $\mu=0.259$ & $\mu=0.500$ & $\mu=0.707$ & $\mu=0.866$ & $\mu=0.940$ & $\mu=0.966$ & $\mu=0.985$ & $\mu=0.996$ & $\mu=1.000$ \\
\hline ke & $0^{\circ}$ & $15^{\circ}$ & $30^{\circ}$ & $45^{\circ}$ & $60^{\circ}$ & $70^{\circ}$ & $75^{\circ}$ & $80^{\circ}$ & $85^{\circ}$ & $90^{\circ}$ \\
\hline 0.25 & 0.0 & 0.0 & 0.0 & 0.0 & -0.1 & & -0.1 & & & -0.2 \\
\hline 0.35 & 0.0 & 0.0 & -0.1 & -0.1 & -0.2 & & 0.3 & & & 0.2 \\
\hline 0.50 & 0.2 & 0.2 & 0.2 & 0.4 & 0.2 & & 0.1 & & & -0.2 \\
\hline 0.71 & 0.3 & 0.3 & 0.4 & 0.4 & 0.3 & & -0.1 & & & -0.2 \\
\hline 1.00 & 0.2 & 0.2 & 0.2 & 0.3 & 0.2 & & -0.1 & & & -0.4 \\
\hline 1.41 & -0.2 & -0.2 & 0.0 & 0.1 & 0.1 & & 0.0 & & & -0.4 \\
\hline 2.00 & -0.6 & -0.6 & -0.4 & -0.2 & 0.0 & & 0.1 & & & -0.2 \\
\hline 2.83 & -0.2 & -0.2 & -0.6 & -0.6 & -0.3 & & -0.1 & & & -0.2 \\
\hline 4.00 & 0.2 & 0.1 & -0.2 & -0.4 & -0.3 & & -0.1 & & & -0.2 \\
\hline 5.66 & -0.1 & 0.0 & 0.0 & -0.1 & -0.3 & & -0.1 & & & -0.1 \\
\hline 8.00 & 0.0 & 0.0 & 0.1 & -0.1 & -0.2 & & -0.1 & & & -0.1 \\
\hline 11.31 & 0.0 & 0.0 & 0.0 & 0.1 & 0.0 & & -0.1 & & & -0.1 \\
\hline 16.00 & 0.0 & 0.0 & 0.0 & 0.0 & -0.1 & & -0.2 & & & 0.0 \\
\hline 22.63 & 0.0 & 0.0 & 0.0 & 0.0 & 0.1 & & -0.2 & & & 0.0 \\
\hline 32.00 & 0.0 & 0.0 & 0.0 & 0.0 & 0.0 & -0.1 & 0.0 & -0.1 & 0.1 & 0.0 \\
\hline 45.25 & 0.0 & 0.0 & 0.0 & 0.0 & 0.0 & & 0.0 & & & 0.0 \\
\hline 64.00 & 0.0 & 0.0 & 0.0 & 0.0 & 0.0 & & -0.1 & 0.0 & 0.0 & 0.1 \\
\hline
\end{tabular}

part than the formula obtained in this paper by combining the high and low frequency approximations from Thomasson (1982). The method from Davy (2009) does not predict the imaginary part of the impedance.

Tables IV and V show the amount in decibels by which the combined approximate method developed in this paper was greater than numerical MATLAB calculations for the imaginary and real parts of the specific radiation wave impedance of a square panel of side length $2 e$ when $\mu \geq 1$. The tabulated data is presented for values of $k e$ in half octave steps from 0.25 to 11.31 and values of $\mu$ in one tenth of a decade steps from 1 to 10 . For the imaginary part, the differences are between 0.8 and $-1.3 \mathrm{~dB}$ with a mean of $0.02 \mathrm{~dB}$ and a standard deviation of $0.28 \mathrm{~dB}$. The real part shows some oscillatory behavior with differences between 6.2 and $-2.4 \mathrm{~dB}$ with a mean of $0.12 \mathrm{~dB}$ and a standard deviation of $1.09 \mathrm{~dB}$. The biggest differences occur when the values are very small and thus would not normally be of any practical importance.

\section{CONCLUSION}

A combined approximation method for calculating both the real and the imaginary parts of the single sided normalized specific forced radiation wave impedance of a finite rectangular panel has been derived. For the real part, the approximate method is between $0.7 \mathrm{~dB}$ higher and $-1 \mathrm{~dB}$ lower than numerical calculations, when the ratio of the transverse wave number in the panel to the wave number in the medium surrounding the panel $\mu$ is less than or equal to one. For the imaginary part, the approximate method is between $2.3 \mathrm{~dB}$ higher and $-2.6 \mathrm{~dB}$ lower than numerical calculations when $\mu$ is less than or equal to one. The method for the real part when $\mu$ is less than or equal to one is not quite as good as the approximate method for the real part when $\mu$ is less than or equal to one developed previously by Davy (2009) which is between $0.4 \mathrm{~dB}$ higher and $-0.6 \mathrm{~dB}$ lower than the numerical calculations. However, unlike the

TABLE IV. Difference in decibels between the combined approximate method developed in this paper and numerical calculations for the imaginary part of the specific forced radiation wave impedance of a square panel of side length $2 e$ when $\mu \geq 1$.

\begin{tabular}{|c|c|c|c|c|c|c|c|c|c|c|c|}
\hline & $\mu=1.00$ & $\mu=1.26$ & $\mu=1.58$ & $\mu=2.00$ & $\mu=2.51$ & $\mu=3.16$ & $\mu=3.98$ & $\mu=5.01$ & $\mu=6.31$ & $\mu=7.94$ & $\mu=10.00$ \\
\hline \multicolumn{12}{|l|}{ ke } \\
\hline 0.25 & 0.1 & 0.2 & 0.2 & 0.3 & 0.4 & 0.5 & 0.8 & 0.5 & 0.1 & -0.1 & 0.0 \\
\hline 0.35 & 0.3 & 0.3 & 0.2 & 0.3 & 0.3 & 0.4 & 0.4 & 0.0 & -0.1 & 0.0 & 0.1 \\
\hline 0.50 & 0.5 & 0.4 & 0.4 & 0.3 & 0.3 & 0.4 & 0.0 & 0.0 & 0.1 & 0.1 & 0.0 \\
\hline 0.71 & 0.6 & 0.5 & 0.4 & 0.4 & 0.3 & 0.1 & 0.0 & 0.0 & 0.0 & 0.0 & 0.0 \\
\hline 1.00 & -0.1 & -0.1 & -0.1 & 0.0 & 0.1 & -0.1 & 0.0 & 0.0 & 0.0 & 0.0 & 0.0 \\
\hline 1.41 & -1.4 & -1.3 & -0.9 & -0.1 & -0.1 & 0.1 & 0.0 & 0.0 & 0.0 & 0.0 & 0.0 \\
\hline 2.00 & -1.0 & -1.1 & -0.6 & -0.1 & 0.1 & 0.0 & 0.0 & 0.0 & 0.0 & 0.0 & 0.0 \\
\hline 2.83 & -0.7 & -0.9 & -0.2 & 0.0 & 0.0 & 0.0 & 0.0 & 0.0 & 0.0 & 0.0 & 0.0 \\
\hline 4.00 & -0.5 & -0.8 & 0.0 & 0.0 & 0.0 & 0.0 & 0.0 & 0.0 & 0.0 & 0.0 & 0.0 \\
\hline 5.66 & -0.3 & -0.6 & 0.1 & 0.0 & 0.0 & 0.0 & 0.0 & 0.0 & 0.0 & 0.0 & 0.0 \\
\hline 8.00 & -0.2 & -0.1 & 0.0 & 0.0 & 0.0 & 0.0 & 0.0 & 0.0 & 0.0 & 0.0 & 0.0 \\
\hline 11.31 & -0.2 & 0.1 & 0.0 & 0.0 & 0.0 & 0.0 & 0.0 & 0.0 & 0.0 & 0.0 & 0.0 \\
\hline
\end{tabular}


TABLE V. Difference in decibels between the combined approximate method developed in this paper and numerical calculations for the real part of the specific forced radiation wave impedance of a square panel of side length $2 e$ when $\mu \geq 1$.

\begin{tabular}{|c|c|c|c|c|c|c|c|c|c|c|c|}
\hline & $\mu=1.00$ & $\mu=1.26$ & $\mu=1.58$ & $\mu=2.00$ & $\mu=2.51$ & $\mu=3.16$ & $\mu=3.98$ & $\mu=5.01$ & $\mu=6.31$ & $\mu=7.94$ & $\mu=10.00$ \\
\hline \multicolumn{12}{|l|}{ ke } \\
\hline 0.25 & 0.1 & 0.1 & 0.1 & 0.1 & 0.2 & 0.4 & 0.8 & -0.3 & -1.9 & -2.4 & -0.8 \\
\hline 0.35 & 0.2 & 0.1 & 0.0 & -0.2 & -0.3 & -0.3 & -0.9 & -2.2 & -2.0 & 1.5 & 6.2 \\
\hline 0.50 & 0.3 & 0.1 & -0.1 & -0.3 & -0.6 & -1.3 & -2.2 & -1.0 & 4.3 & 1.3 & -2.4 \\
\hline 0.71 & 0.4 & 0.2 & 0.1 & -0.2 & -0.9 & -1.8 & 0.3 & 4.0 & -1.3 & -0.4 & 0.9 \\
\hline 1.00 & 0.5 & 0.5 & 0.5 & 0.2 & -1.3 & 0.8 & 1.6 & -1.5 & 1.9 & -1.4 & 1.5 \\
\hline 1.41 & 0.7 & 0.8 & 1.0 & -0.3 & 0.3 & 0.8 & -0.8 & 0.8 & -0.4 & -0.4 & -0.3 \\
\hline 2.00 & 0.8 & 1.0 & 1.0 & -0.3 & 1.2 & -1.0 & 1.0 & -0.3 & -0.8 & -0.7 & 0.6 \\
\hline 2.83 & 0.6 & 0.9 & 0.0 & 1.1 & -0.7 & 0.3 & 0.5 & 0.4 & 0.5 & -0.4 & -0.4 \\
\hline 4.00 & 0.5 & 1.0 & 0.7 & -0.7 & 0.5 & 0.6 & 0.6 & -0.3 & 0.6 & 0.5 & -0.1 \\
\hline 5.66 & 0.3 & 1.2 & 0.3 & 0.7 & 0.5 & 0.3 & -0.3 & -0.4 & 0.2 & 0.0 & -0.3 \\
\hline 8.00 & 0.3 & 0.0 & -0.3 & -0.1 & 0.3 & -0.1 & 0.0 & -0.1 & 0.0 & 0.1 & 0.1 \\
\hline 11.31 & 0.2 & 0.9 & -0.1 & 0.1 & -0.1 & 0.2 & 0.1 & -0.1 & 0.0 & 0.1 & -0.1 \\
\hline
\end{tabular}

method from Davy (2009), the method developed in this paper can also calculate the imaginary part.

For the imaginary part, the approximate method is between $0.8 \mathrm{~dB}$ higher and $-1.3 \mathrm{~dB}$ lower than numerical calculations when $\mu$ is greater than or equal to one. For the real part, the approximate method is between $6.2 \mathrm{~dB}$ higher and $-2.4 \mathrm{~dB}$ lower than numerical calculations, when $\mu$ is greater than or equal to one.

Allard, J. F., and Atalla, N. (2009). Propagation of Sound in Porous Media: Modelling Sound Absorbing Materials (John Wiley and Sons, Chichester, UK), pp. 1-358.

Brunskog, J. (2012). "The forced sound transmission of finite single leaf walls using a variational technique," J. Acoust. Soc. Am. 132, 1482-1493.

Cremer, L., Heckl, M., and Petersson, B. A. T. (2005). Structure-Borne Sound-Structural Vibrations and Sound Radiation at Audio Frequencies 3rd ed. (Springer, Berlin), pp. 1-607.

Davy, J. L. (2009). "The forced radiation efficiency of finite size flat panels that are excited by incident sound," J. Acoust. Soc. Am. 126, 694-702.

Fahy, F. (1985). Sound and Structural Vibration—Radiation, Transmission and Response (Academic Press, London), pp. 1-309.

Gösele, K. (1953). "Schallabstrahlung von Platten, die zu Biegeschwingungen angeregt sind" ("Sound-radiating plates which are excited to bending vibrations"), Acustica 3, 243-248.

Gradshteyn, I. S., and Ryzhik, I. M. (1965). Table of Integrals, Series, and Products, 4th ed., edited by A. Jeffrey (Academic Press, New York), pp. $1-1086$.

Jeong, C.-H. (2013). "Converting Sabine absorption coefficients to random incidence absorption coefficients," J. Acoust. Soc. Am. 133, 3951-3962.

Leppington, F. G., Broadbent, E. G., and Heron, K. H. (1982). "The acoustic radiation efficiency of rectangular panels," Proc. R. Soc. London, Ser. A 382, 245-271.

Li, W. L., and Gibeling, H. J. (2000). "Determination of the mutual radiation resistances of a rectangular plate and their impact on the radiated sound power," J. Sound. Vib. 229, 1213-1233.
Lindblad, S. G. (1973). Akustik II Compendium (Acoustics II Compendium), (Department of Building Acoustics, University of Lund, Lund, Sweden), pp. 1-90.

Lindblad, S. G. (1985). Akustik IV (Acoustics IV) (Department of Building Acoustics, University of Lund, Lund, Sweden), pp. 1-163.

Ljunggren, S. (1991). "Airborne sound insulation of thin walls," J. Acoust. Soc. Am. 89, 2324-2337.

Lyon, R. H., and Maidanik, G. (1962). "Power flow between linearly coupled oscillators," J. Acoust. Soc. Am. 34, 623-639.

Maidanik, G. (1962). "Response of ribbed panels to reverberant acoustic fields," J. Acoust. Soc. Am. 34, 809-826.

Maidanik, G. (1975). "Erratum: Response of ribbed panels to reverberant acoustic fields," J. Acoust. Soc. Am. 57, 1552.

Morse, P. M., and Ingard, K. U. (1968). Theoretical Acoustics (McGrawHill, New York), pp. 1-927.

Novak, R. A. (1992). "Sound insulation of lightweight double walls," Appl. Acoust. 37, 281-303.

Novak, R. A. (1995). "Radiation from partially excited plates," Acta Acust. 3, 561-567.

Rindel, J. H. (1975). "Transmission of traffic noise through windowsInfluence of incident angle on sound insulation in theory and experiment," Report No. 9, The Acoustics Laboratory, Technical University of Denmark, Lyngby, Denmark, pp. 1-240.

Rindel, J. H. (1993a). "Modelling the angle-dependent pressure reflection factor," Appl. Acoust. 38, 223-234.

Rindel, J. H. (1993b). "Sound transmission through single layer walls," in Noise-93, Proceedings of the International Conference on Noise and Vibration Control, edited by M. J. Crocker and N. I. Ivanov (St. Petersburg, Russia), pp. 63-68.

Sato, H. (1973). "On the mechanism of outdoor noise transmission through walls and windows-A modification of infinite wall theory with respect to radiation of transmitted wave," J. Acoust. Soc. Jpn. 29, 509-516.

Stenzel, H. (1952). "Die akustische Strahlung der rechteckigen Kolbenmembran" ("The acoustic radiation of a rectangular piston diaphragm"), Acustica 2, 263-281.

Thomasson, S.-I. (1980). "On the absorption coefficient," Acust. 44, 265-273.

Thomasson, S.-I. (1982). "Theory and experiments on the sound absorption as function of the area," Report No. TRITA-TAK-8201, Department of Technical Acoustics, Royal Institute of Technology, Stockholm, Sweden, pp. 1-68. 